\title{
MATROIDS WHOSE GROUND SETS ARE DOMAINS OF FUNCTIONS
}

\author{
JAMES OXLEY, KEVIN PRENDERGAST and DON ROW
}

(Received 13 October 1980)

Communicated by W. D. Wallis

\begin{abstract}
From an integer-valued function $f$ we obtain, in a natural way, a matroid $M_{f}$ on the domain of $f$. We show that the class $\mathscr{R}$ of matroids so obtained is closed under restriction, contraction, duality, truncation and elongation, but not under direct sum. We give an excluded-minor characterization of $\mathscr{T}$ and show that $\mathfrak{K}$ consists precisely of those transversal matroids with a presentation in which the sets in the presentation are nested. Finally, we show that on an $n$-set there are exactly $2^{n}$ members of Tr.
\end{abstract}

1980 Mathematics subject classification (Amer. Math. Soc.): 05 B 35.

\section{Introduction}

From any function $f: E \rightarrow Z$, where $E$ is a finite set and $Z$ is the set of integers, we obtain a function with domain $2^{E}$ whose value at $A \subseteq E$ is $\max \{f(a) \mid a \in A\}$ if $A$ is non-empty and $\min \{f(a) \mid a \in E\}$ otherwise. This function is semimodular and increasing and as in Chapter 7 of Crapo and Rota (1970) we obtain a matroid $M_{f}$ whose independent sets are exactly the subsets $I$ of $E$ such that $\max \{f(a) \mid a$ $\in J\} \geqslant|J|$ for all non-empty subsets $J$ of $I$. This paper investigates the class $\mathfrak{K}$ of matroids obtained in this way. Firstly, we show that $9 \pi$ consists exactly of those matroids, all of whose minors are free or have a unique minimal non-trivial flat. Secondly, we give an excluded minor characterisation of $\mathfrak{N}$. In obtaining this we prove $\pi$ closed under duality. Finally, we show that the members of $\pi$ are transversal and we use a result of Welsh (1969) to count the members of $M$.

CCopyright Australian Mathematical Society 1982 
In general, we follow Welsh (1976) for matroid terminology. The ground set of a matroid $M$ will be denoted by $E(M)$ or just $E$. If $T \subseteq E$, we shall sometimes write $M \backslash T$ and $M / T$ for, respectively, the restriction and contraction of $M$ to $E \backslash T$. The rank and closure of $T$ in $M$ will be denoted by $\operatorname{rk}(T)$ and $\sigma(T)$ respectively, and the subscript "cont" will be added to distinguish the rank and closure in a contraction of $M$. A flat $F$ in $M$ is non-trivial provided $F$ is dependent. We call $F$ a non-trivial extension of a flat $H$ if $H \subseteq F$ and $F \backslash H$ is a non-trivial flat in $M / H$; otherwise, $F$ is called a free extension of $H$. Except where otherwise stated, if $|E|=n$, we will identify $E$ with the set $\{1,2, \ldots, n\}$ in such a way that if $i<j$, then $f(i) \leqslant f(j)$.

We use the following properties of $\Re$.

LEMMA 1. For any member $M_{f}$ of $\Re$,

(i) $I=\left\{i_{1}, i_{2}, \ldots, i_{s}\right\}$, with $i_{1}<i_{2}<\cdots<i_{s}$, is independent in $M_{f}$ exactly when $f\left(i_{r}\right) \geqslant r$ for all $r=1,2, \ldots, s$

(ii) $I=\left\{i_{1}, i_{2}, \ldots, i_{s}\right\}$, with $i_{1}<i_{2}<\cdots<i_{s}$, is independent in $M_{f}$ exactly when $|I \cap\{1,2, \ldots, r\}| \leqslant f(r)$ for all $r=1,2, \ldots, n$;

(iii) $C=\left\{c_{1}, c_{2}, \ldots, c_{s}\right\}$, with $c_{1}<c_{2}<\cdots<c_{s}$, is a circuit in $M_{f}$ exactly when $s-1 \geqslant f\left(c_{r}\right) \geqslant \min \{r, s-1\}$ for all $r=1,2, \ldots, s$;

(iv) for each $e$ in a minimal non-trivial flat $F$ in $M_{f}, f(e) \leqslant \operatorname{rk}(F)$.

Proof. (i) If $i_{r}$ is the maximal element of a subset $J$ of $I$, then $r \geqslant|J|$ and so, if $f\left(i_{r}\right) \geqslant r$, then $\max \{f(x) \mid x \in J\} \geqslant|J|$. Conversely, if $I$ is independent, $\left\{i_{1}, i_{2}, \ldots, i_{r}\right\} \subseteq I$ ensures $f\left(i_{r}\right) \geqslant r$.

(ii) For any $k$, let $r_{0}$ be the minimum $r$ for which $\{1,2, \ldots, r\} \cap I=$ $\left\{i_{1}, i_{2}, \ldots, i_{k}\right\}$. Then $i_{k}=r_{0}$ and, if $k \leqslant f\left(r_{0}\right)$, we have $f\left(i_{k}\right)=f\left(r_{0}\right) \geqslant k$ and $I$ is independent by (i). Conversely, if $I$ is independent then, by (i), $f\left(i_{r}\right) \geqslant r \geqslant \mid I \cap$ $\{1,2, \ldots, r\} \mid$.

(iii) As $s-1 \geqslant f\left(c_{r}\right) \geqslant \min \{r, s-1\}$ for all $r=1,2, \ldots, s$, we have $\max \{f(x) \mid x \in C\}=s-1$ and so $C$ is dependent. But any non-empty subset $P \subset C$ of size $r$ contains an element $c \geqslant c_{r}$ and so $\max \{f(x) \mid x \in P\} \geqslant r$. Hence each proper subset of $C$ is independent. Conversely, if $C$ is a circuit, then $\left\{c_{1}, c_{2}, \ldots, c_{r}\right\}$ is independent for $r<s$ and so, by (i), $f\left(c_{r}\right) \geqslant r$, but as $C$ is dependent $f\left(c_{r}\right) \leqslant f\left(c_{s}\right)<s$.

(iv) As $e$ is in some circuit $C^{\prime} \subseteq F$, we have by (iii), $f(e) \leqslant\left|C^{\prime}\right|-1=\operatorname{rk}\left(C^{\prime}\right) \leqslant$ $\operatorname{rk}(F)$.

\section{Characterisation by flats}

We denote by $\mathfrak{K}^{\prime}$ the class of matroids having the property that each minor is either a free matroid or has a unique minimal non-trivial flat. 
LEMMA 2. Each member $M$ of $\mathfrak{T}^{\prime}$ has a finite chain $\sigma(\varnothing)=F_{0} \subset F_{1} \subset \cdots \subset$ $F_{k} \subseteq E$ of flats where either $F_{i+1}$ is the unique minimal non-trivial extension of $F_{i}$ or $F_{i+1}=E$, the latter holding when $F_{i}$ has no non-trivial extensions. Each flat in $M$ is a direct sum of some $F_{i}$ and a free matroid.

Proof. Unless $M$ is free it has a unique minimal non-trivial flat. If $F_{0}=\sigma(\varnothing)$ is empty we write $F_{1}$ for this minimal non-trivial flat, otherwise it is $F_{0}$. Let us suppose that the chain $\sigma(\varnothing)=F_{0} \subset F_{1} \subset \cdots \subset F_{i}$ exists as required. Then either there is a unique minimal non-trivial extension $F_{i+1}$ of $F_{i}$ or not. If not, either $E$ is a free extension of $F_{i}$, or $M$ has two minimal non-trivial extensions $H, H^{\prime}$ of $F_{i}$. In the latter case, since $H \cap H^{\prime}$ is a flat in $M$, both $H \backslash H^{\prime}$ and $H^{\prime} \backslash H$ are flats in $M /\left(H \cap H^{\prime}\right)$. But $H$ and $H^{\prime}$ are non-trivial extensions of $F_{i}$ in $M$ and $M /\left(H \cap H^{\prime}\right) \in \mathfrak{T}^{\prime}$, so there is a unique minimal non-trivial flat of $M /\left(H \cap H^{\prime}\right)$ contained in both $H \backslash H^{\prime}$ and $H^{\prime} \backslash H$, contradicting $\left(H \backslash H^{\prime}\right) \cap\left(H^{\prime} \backslash H\right)=\varnothing$. Thus we inductively obtain the required chain of flats. For any flat $F$ in $M$ there is a maximal $i \leqslant k$ such that $F_{i} \subseteq F$. Then $F$ is a free extension of $F_{i}$, that is, a direct sum of $F_{i}$ and the free matroid $M \mid\left(F \backslash F_{i}\right)$.

We prove $\mathscr{R} \supseteq \mathfrak{R}^{\prime}$ by characterising the circuits of members of $\mathfrak{R}^{\prime}$. In the next two results, the flats $F_{0}, F_{1}, \ldots, F_{k}$ are as specified in the preceding lemma.

LEMMA 3. For any $M \in \Re^{\prime}$ the circuits contained in $F_{i}$ but not in $F_{i-1}$ are exactly the sets $C$ satisfying $|C|=\operatorname{rk}\left(F_{i}\right)+1$ and $\left|C \cap F_{j}\right| \leqslant \operatorname{rk}\left(F_{j}\right)$ for all $j<i$.

Proof. Again we proceed by induction. Either $F_{0}=\varnothing$ or each element of $F_{0}$ is a loop $C$ satisfying $|C|=1=$ rk $F_{0}+1$. Now suppose the circuits contained in $F_{j}$ but not $F_{j-1}$ are as prescribed for all $j<i$. If $C$ is a circuit contained in $F_{i}$ but not $F_{i-1}$, then $\sigma(C)$ is a non-trivial flat, every element of which is in a circuit. From the previous lemma, $\sigma(C)=F_{j}$, for some $j$. Consequently $\sigma(C)=F_{i}$, and $|C|=$ $\operatorname{rk}\left(F_{i}\right)+1$. For $j<i$, if $C \cap F_{j} \neq C$, then $C \cap F_{j}$ is independent and so $\left|C \cap F_{j}\right|$ $=\operatorname{rk}\left(C \cap F_{j}\right) \leqslant \operatorname{rk}\left(F_{j}\right)$. Thus every circuit of $M$ is of the specified form. Conversely, suppose $C$ is contained in $F_{i}$ but not $F_{i-1},|C|=\operatorname{rk}\left(F_{i}\right)+1$ and $\left|C \cap F_{j}\right|$ $\leqslant \operatorname{rk}\left(F_{j}\right)$ for all $j<i$. As $C \subseteq F_{i}$ it is dependent and so contains a circuit $C^{\prime}$. If $C^{\prime} \subseteq F_{j}$ for some $j<i,\left|C^{\prime}\right|=\left|C^{\prime} \cap F_{j}\right| \leqslant\left|C \cap F_{j}\right| \leqslant \operatorname{rk}\left(F_{j}\right)$. Thus $\left|C^{\prime}\right| \neq \operatorname{rk}\left(F_{j}\right)$ +1 , contradicting the proven property of any such circuit. Hence $\left|C^{\prime}\right|=\operatorname{rk}\left(F_{i}\right)$ $+1=|C|$, and $C=C^{\prime}$, a circuit. We have inductively characterised all circuits contained in some $F_{i}$. But $E$ is a free extension of $F_{k}$ and so any circuit in $E$ is also in $F_{k}$.

LEMMA 4. $\cong \supseteq \Re^{\prime}$. 
Proof. For $M \in \Re^{\prime}$, letting $F_{-1}=\varnothing$, we define an appropriate function on the underlying set $E$ of $M$ as follows:

$$
f(e)= \begin{cases}\operatorname{rk}\left(F_{i}\right) & \text { if } e \in F_{i} \backslash F_{i-1} \text { for some } i \geqslant 0 \\ \operatorname{rk}(E) & \text { if } e \in E \backslash F_{k}\end{cases}
$$

We prove $M_{f}=M$ by considering the circuits in both. If $C$ is a circuit in $M$ then, for some $i \geqslant 0, C \subseteq F_{i}, C \nsubseteq F_{i-1},|C|=\operatorname{rk}\left(F_{i}\right)+1$ and $\left|C \cap F_{j}\right| \leqslant \operatorname{rk}\left(F_{j}\right)$ for all $j<i$. Let $C=\left\{c_{1}, c_{2}, \ldots, c_{s}\right\}$ with $c_{1}<c_{2}<\cdots<c_{s}$. Then for all $r, f\left(c_{r}\right) \leqslant$ $f\left(c_{s}\right)=\operatorname{rk}\left(F_{i}\right)=|C|-1=s-1$. On the other hand, either $c_{r} \in$ $F_{i} \backslash F_{i-1}$, for some $j<i$. In the first case, $f\left(c_{r}\right)=\operatorname{rk}\left(F_{i}\right)=s-1$, and in the second case $f\left(c_{r}\right)=\operatorname{rk}\left(F_{j}\right) \geqslant\left|C \cap F_{j}\right| \geqslant r$. We conclude that $s-1 \geqslant f\left(c_{r}\right) \geqslant$ $\min \{r, s-1\}$ for all $r=1,2, \ldots, s$ and so, by Lemma 1(iii), $C$ is a circuit in $M_{f}$. Conversely, if $C$ is a circuit in $M_{f}$ the above pair of inequalities hold for each $c_{r} \in C$, and so $f\left(c_{s}\right)=s-1=\operatorname{rk}\left(F_{i}\right)$, say. Then for all $j<i, C \cap F_{j}=\left\{c_{r} \mid f\left(c_{r}\right)\right.$ $\left.\leqslant \operatorname{rk}\left(F_{j}\right)\right\} \subseteq\left\{c_{r} \mid r \leqslant \operatorname{rk}\left(F_{j}\right)\right\}$, giving $\left|C \cap F_{j}\right| \leqslant \operatorname{rk}\left(F_{j}\right)$. But $s=|C|=\operatorname{rk}\left(F_{i}\right)+1$. Hence $C$ is a circuit in $M$.

In view of Lemma 4 , to prove $\mathfrak{K}^{\prime}=\mathscr{K}$ it suffices to show that $\mathscr{N}$ is closed with respect to taking minors and that each $M_{f} \in \mathfrak{R}$ is a free matroid or has a unique minimal non-trivial flat.

LEMMA 5. Each $M_{f} \in \mathfrak{R}$ is a free matroid or has a unique minimal non-trivial flat.

Proof. Let $H$ and $H^{\prime}$ be distinct minimal non-trivial flats in $M_{f}$ with $\mathrm{rk}(H) \leqslant$ $\operatorname{rk}\left(H^{\prime}\right)$. For $e \in H \backslash H^{\prime}$, by Lemma $1(\mathrm{iv}), f(e) \leqslant \mathrm{rk}(H)$. For any maximal independent subset $I$ of $H^{\prime}, I \cup e$ is independent. But $\max \{f(x) \mid x \in I \cup e\} \leqslant$ $\max \left\{\operatorname{rk}\left(H^{\prime}\right), \operatorname{rk}(H)\right\}=\operatorname{rk}\left(H^{\prime}\right)<|I \cup e|$, contradicting the independence of $I \cup$ $e$. Thus $H \subseteq H^{\prime}$ and $H=H^{\prime}$.

LEMMA 6. Any restriction of a member of $\mathfrak{T}$ is also in $\mathfrak{R}$.

Proof. Clearly if $f: E \rightarrow Z$ defines $M_{f}$, then $\left.f\right|_{T}$ defines $M_{f} \mid T$.

In order to show $\Re$ closed with respect to taking contractions we prove $\mathscr{R}$ closed under duality. We call a function $f: E \rightarrow Z$ a standard function if $f(1)=0$ or 1 , and $0 \leqslant f(r+1)-f(r) \leqslant 1$ for all $r=1,2, \ldots, n-1$.

LEMMA 7. Any matroid $M_{f}$ is defined by a standard function. 
Proof. Define

$$
g(1)=\left\{\begin{array}{ll}
1 & \text { if } f(1) \geqslant 1, \\
0 & \text { otherwise, }
\end{array} \quad g(r+1)= \begin{cases}g(r)+1 & \text { if } f(r+1)>g(r), \\
g(r) & \text { otherwise, }\end{cases}\right.
$$

for $r=1,2, \ldots, n-1$. Using induction on $r$, commencing with $r_{0}$, the least $r$ for which $f(r) \geqslant 0$, we see that $f(r) \geqslant g(r)$. Consequently any independent set $I=\left\{i_{1}, i_{2}, \ldots, i_{s}\right\}$ in $M_{g}$ has $i_{1} \geqslant r_{0}$ and so $f\left(i_{r}\right) \geqslant g\left(i_{r}\right) \geqslant r$, ensuring $I$ independent in $M_{f}$. Conversely, suppose $I$ is independent in $M_{f}$. Then $f\left(i_{1}\right) \geqslant 1$ and so if either $i_{1}=1$ or $i_{1}>1$ we have $g\left(i_{1}\right) \geqslant 1$. Assuming $g\left(i_{r}\right) \geqslant r$ we consider $g\left(i_{r+1}\right)$. Either $g\left(i_{r+1}\right)>g\left(i_{r+1}-1\right)$ and $g\left(i_{r+1}-1\right) \geqslant g\left(i_{r}\right) \geqslant r$ giving $g\left(i_{r+1}\right) \geqslant r+1$, or $g\left(i_{r+1}\right)=g\left(i_{r+1}-1\right)$ and $f\left(i_{r+1}\right) \leqslant g\left(i_{r+1}-1\right)$ giving $g\left(i_{r+1}\right)=f\left(i_{r+1}\right) \geqslant r$ +1 . In both cases we have $g\left(i_{r}\right) \geqslant r$, for all $r=1,2, \ldots, s$ by induction. Consequently $I$ is independent in $M_{g}$.

LEMMA 8. $\left(M_{f}\right)^{*}$ is in $\mathfrak{T}$.

Proof. We may assume $f$ is a standard function. Let $m=\operatorname{rk}(E)$. Then $m=f(n)$. We prove that if $f^{*}: E \rightarrow Z$ is defined by $f^{*}(1)=n-m, f^{*}(r+1)=n$ $-m+f(r)-r$, for all $r=1,2, \ldots, n-1$, then $\left(M_{f}\right)^{*}=M_{f^{*}}$. Now let $B$ be an $m$-element subset of $E$. Then it is routine to check that each statement in the following list is equivalent to its predecessor. The equivalence of (v) and (vi) uses the fact that $f(n)=m$ and $f^{*}(1)=n-m$, and the equivalence of (vi) and (vii) uses Lemma 1 and the fact that $f^{*}$ is monotonic non-increasing.

(i) $B$ is a base of $M_{f}$;

(ii) $|B \cap\{1,2, \ldots, r\}| \leqslant f(r)$ for all $r=1,2, \ldots, n$;

(iii) $|B \cap\{r+1, r+2, \ldots, n\}| \geqslant m-f(r)$ for all $r=1,2, \ldots, n$;

(iv) $|B \cap\{n-r+1, n-r+2, \ldots, n\}| \geqslant m-f(n-r)$ for all $r=0,1, \ldots, n$ -1 ;

(v) $|(E \backslash B) \cap\{n-r+1, n-r+2, \ldots, n\}| \leqslant r-m+f(n-r)$ for all $r=$ $0,1, \ldots, n-1$;

(vi) $|(E \backslash B) \cap\{n-r+1, n-r+2, \ldots, n\}| \leqslant f^{*}(n-r+1)$ for all $r=$ $1,2, \ldots, n$;

(vii) $E \backslash B$ is a base of $M_{f^{*}}$.

LEMMA 9. Any contraction of a member of $\Re$ is in $\Re$.

ProOF. $M_{f} \cdot T=\left(M_{f}^{*} \mid T\right)^{*}$.

THEOREM 10. $\mathscr{M}=\mathscr{K}^{\prime}$. 


\section{Excluded minor characterisation}

We characterise $\Re^{\prime}$, and hence $\Re$, by its excluded minors. For $k=2,3, \ldots$, consider a set $E$ which is the disjoint union of two $k$-element subsets $E_{1}$ and $E_{2}$ and put $\mathcal{C}=\left\{E_{1}, E_{2}\right\} \cup\left\{C\left|C \not \supset E_{1}, C \not \supset E_{2}, C \subset E,\right| C \mid=k+1\right\}$.

LEMma 11. For each $k=2,3, \ldots, \mathcal{C}$ is the collection of circuits of a matroid $N^{k}$ on $E$.

Proof. Consider any two distinct members $C_{1}, C_{2}$ of $\mathcal{C}$ with a common element $e$. Then $\left|\left(C_{1} \cup C_{2}\right) \backslash e\right| \geqslant k+1$ and so $\left(C_{1} \cup C_{2}\right) \backslash e$ contains a member of $e$.

LEMMA 12. $N^{k} \notin \mathfrak{T}^{\prime}$.

Proof. Both $E_{1}$ and $E_{2}$ are minimal non-trivial flats.

THEOREM 13. $\mathfrak{R}^{\prime}$ is the class of matroids having no minor isomorphic to $N^{k}$ for $k=2,3, \ldots$.

Proof. Suppose that $M$ is not in $\mathfrak{N}^{\prime}$ but every proper minor of $M$ is in 'N'. Then $M$ has two minimal non-trivial flats $E_{1}$ and $E_{2}$, say. If $E \neq E_{1} \cup E_{2}$, choose $e \in E \backslash\left(E_{1} \cup E_{2}\right)$ and consider $M \backslash e$. In this restriction both $E_{1}$ and $E_{2}$ are still minimal non-trivial flats, contradicting the choice of $M$. Thus $E=E_{1} \cup E_{2}$.

We now show that each of $E_{1}$ and $E_{2}$ is a circuit of $M$. If $E_{1}$ is not, then $M$ has a circuit $C \subset E_{1}$. Choose $e \in E_{1} \backslash C$ and consider the contraction $M / e$. Again $E_{1} \backslash e$ and $E_{2} \backslash e$ are minimal non-trivial flats in $M / e$, contradicting our choice of $M$. Thus $E_{1}$, and similarly $E_{2}$, is a circuit of $M$.

We now prove $E_{1}$ and $E_{2}$ are disjoint. If not, choose $e \in E_{1} \cap E_{2}$. In $M / e$ both $E_{1} \backslash e$ and $E_{2} \backslash e$ are flats and circuits, and so are minimal non-trivial flats. Thus $E_{1} \backslash e=E_{2} \backslash e$ ensuring $E_{1}=E_{2}$, contradicting our initial choice of $E_{1}$ and $E_{2}$. So $E=E_{1} \cup E_{2}$.

Next we prove $\left|E_{1}\right|=\left|E_{2}\right|$. Suppose to the contrary that $\left|E_{1}\right|<\left|E_{2}\right|$, that is, $\operatorname{rk}\left(E_{1}\right)<\operatorname{rk}\left(E_{2}\right)$. Choosing $e \in E_{2}$ we consider the contraction $M / e$. In this contraction $E_{2} \backslash e$ is a circuit and a flat and so is a minimal non-trivial flat of $M / e$. Also $\sigma_{\text {cont }}\left(E_{1}\right)=\sigma\left(E_{1} \cup e\right) \backslash e$ is a non-trivial flat in $M / e$. Thus we have $E_{2} \backslash e \subseteq \sigma_{\text {cont }}\left(E_{1}\right)$. Now $\mathrm{rk}_{\text {cont }}\left(E_{2} \backslash e\right)=\operatorname{rk}\left(E_{2}\right)-1 \geqslant \operatorname{rk}\left(E_{1}\right)$ and $\mathrm{rk}_{\text {cont }}\left(E_{1}\right)=$ $\operatorname{rk}\left(E_{1} \cup e\right)-1=\operatorname{rk}\left(E_{1}\right)$, since $E_{1}$ is a flat in $M$. Hence $\operatorname{rk}_{\text {cont }}\left(E_{2} \backslash e\right) \geqslant$ $\mathrm{rk}_{\text {cont }}\left(E_{1}\right)$. Thus $E_{2} \backslash e=\sigma_{\text {cont }}\left(E_{1}\right)=\sigma\left(E_{1} \cup e\right) \backslash e$, ensuring that, in $M, E_{2}$ contains $E_{1}$. From this contradiction we can assume $\left|E_{1}\right| \geqslant\left|E_{2}\right|$; similarly $\left|E_{2}\right| \geqslant \mid$ $E_{1} \mid$, giving $\left|E_{1}\right|=\left|E_{2}\right|=k$, say, for some $k>1$. 
It now remains only to prove that the other circuits in $M$ are exactly the subsets of $E$ of size $k+1$ which contain neither $E_{1}$ nor $E_{2}$. By supposing that we initially specified $E_{1}$ as a non-trivial flat of minimal rank in $M$ we deduce that each circuit in $M$ has at least $k$ elements. Suppose that $C$ is a third circuit of size $k$ in $M$, then $C \cap E_{1} \neq \varnothing \neq C \cap E_{2}$. Hence $\sigma(C)$ is a minimal non-trivial flat of rank $k-1$ in $M$ and $E_{1} \neq \sigma(C)$. But on proceeding as before with $\sigma(C)$ in place of $E_{2}$ we show $\sigma(C) \cap E_{1}=\varnothing$, contradicting $C \cap E_{1} \neq \varnothing$. So each circuit other than $E_{1}$ or $E_{2}$ has at least $k+1$ elements. We need only show $\operatorname{rk}(M)=k$ to prove all $(k+1)$-element subsets of $E$ dependent and the circuits are as specified. Choosing $e \in E_{2}$ and considering the contraction $M / e$, as above, we have $E_{2} \backslash e \subseteq$ $\sigma\left(E_{1} \cup e\right) \backslash e$, ensuring $E_{2} \subseteq \sigma\left(E_{1} \cup e\right)$ and so $E_{1} \cup e$ spans $M$, $\operatorname{giving} \operatorname{rk}(M)=$ $\operatorname{rk}\left(E_{1} \cup e\right)=\operatorname{rk}\left(E_{1}\right)+1=k$. Consequently $M=N^{k}$, for some $k>1$.

In the preceding section it was shown that $\mathscr{R}$ is closed under restriction, contraction and duality. It is straightforward to check that, in addition, $\mathscr{R}$ is closed under truncation and hence also under elongation. However, $\mathfrak{R}$ is not closed under direct sum, for, although all uniform matroids are in $\Re$, the direct sum of two uniform matroids each having rank and corank at least one has $N^{2}$ as a minor and so is not in $\mathscr{N}$. We now show that $\mathfrak{R}$ is a sub-class of the class of transversal matroids.

THEOREM 14. $A$ matroid $M$ is in $\Re$ if and only if $M$ is the transversal matroid $M\left[\left(A_{i} \mid i \in\{1,2, \ldots, m\}\right)\right]$ of a family $\left(A_{i} \mid i \in\{1,2, \ldots, m\}\right)$ of subsets of a set $E$ where $A_{1} \supseteq A_{2} \supseteq \cdots \supseteq A_{m}$.

Proof. If $M$ is transversal having a presentation of the specified type, then define

$$
f(j)= \begin{cases}0 & \text { if } j \notin A_{1}, \\ i & \text { if } j \in A_{i} \backslash A_{i+1} \\ m & \text { if } j \in A_{m} .\end{cases}
$$

It is routine to check that $M_{f}$ is equal to $M$. Conversely, if $M_{f} \in \mathfrak{R}$, let $A_{i}=\{j \in E \mid f(j) \geqslant i\}$. Then again one can easily check that $M_{f}$ is $M\left[\left(A_{i} \mid i \in\{1,2, \ldots, \operatorname{rk}(M)\}\right)\right]$.

As $\mathscr{T}$ is closed under duality, one can use the Ingleton-Piff construction (see, for example, Welsh (1976), page 221) with the preceding result to obtain a simple representation of a member of $\mathscr{T}$ as a strict gammoid. Moreover, if $M \cong M_{f}$ where $f$ is a standard function, it is not difficult to show that $M^{*}$ is isomorphic to the fundamental transversal matroid associated with the cobase $B$ of $M^{*}$ where 
$B=\left\{i_{1}, i_{2}, \ldots, i_{\mathrm{rk}(M)}\right\}$ with $f\left(i_{j}\right)=j$ for all $j=1,2, \ldots, \operatorname{rk}(M)$. Thus $\mathscr{N}$ is a sub-class of the class of fundamental transversal matroids.

Welsh (1969) gave a lower bound on the number of transversal matroids on an $n$-set $S$ by constructing exactly $2^{n}$ non-isomorphic transversal matroids on $S$. It is straightforward to check that the union over all positive integers $n$ of these sets of matroids is precisely the class $\mathfrak{T}$. Hence, by Theorems 1 and 2 of Welsh (1969), we have that on an $n$-set there are precisely $2^{n}$ non-isomorphic members of $\Re$ and of these exactly $\left(\begin{array}{l}n \\ r\end{array}\right)$ have rank $r$.

\section{References}

H. H. Crapo and G.-C. Rota (1970), On the foundations of combinatorial theory: combinatorial geometries (M.I.T. Press, Cambridge, Massachusetts).

D. J. A. Welsh (1969), 'A bound for the number of matroids', J. Combinatorial Theory 6, 313-316.

D. J. A. Welsh (1976), Matroid theory (Academic Press, London).

Mathematics Department, IAS

Hydro Electric Commission

Australian National University

Hobart

Canberra

Australia

Australia

Mathematics Department

University of Tasmania

Hobart

Australia 